\title{
On Hybrid Control Problems in Communication Systems
}

\author{
Karl Henrik Johansson and Fortunato Santucci
}

\begin{abstract}
The importance of a strong research agenda on the convergence of communications and control has been emphasized by several researchers recently. The purpose of this paper is to evidence through concrete application examples how the envisioned synergy can actually be exploited. The focus is on control of wireless communication networks. In order to address in a rigorous and exhaustive way the complexity of interactions that usually arise in these systems, we try to devise how hybrid modelling may intrinsically provide a theoretical framework to formulate problems and provide partial solutions.
\end{abstract}

\section{INTRODUCTION}

The rapid technologies advances in embedded processors and networking has recently motivated interests and expectations for a large set of applications that rely on networked embedded systems [1]. Embedded processors are widely used in, e.g., automotive, entertainment and communication devices, and in a wide range of appliances. On the other side, networking technologies (especially those based on the wireless medium) have also known a rapid growth, thus paving the way to conceive large sets of (radio) interconnected embedded devices. As micro-fabrication technology advances make it cheaper to build single sensor and actuator nodes, a large set of new applications can be envisaged in environment monitoring, smart agriculture, energy efficient heating, home automation etc. Moreover, a major impact of wireless interconnections can be expected in industrial automation, where updating production lines will not induce anymore expensive and time consuming re-cabling. In summary, we can envisage a networked embedded system as an eventually large set of sensors, controllers and actuators linked via wired and wireless communication channels. While technology advances and prospected applications are progressing, it has to be recognized that developing sound methods for design and operations of such systems is a major research challenge [2], [3]. In fact, traditional control theory typically relies on accurate and lossless feedbacks, with no time delay jitter. On the other side, communication networks are designed for applications that typically are either delay tolerant (e.g., data transfer) or error tolerant

The work was partially supported by European Commission through the Network of Excellence HYCON, by the Swedish Foundation for Strategic Research through an Individual Grant for the Advancement of Research Leaders, and by the Swedish Research Council.

K. H. Johansson is with Department of Signals, Sensors and Systems, Royal Institute of Technology, Stockholm, Sweden. kallejes3.kth.se

F. Santucci is with Center of Excellence DEWS, University of L'Aquila, L'Aquila, Italy. santucci@ing. univaq. it (e.g., for conversational services). Looking at the design problem from the communication side and thus keeping in mind the layered open system interconnection (OSI) model, we can cast the control over network problem as an application to be delivered over an underlying protocol stack.

A control application may require large communication channel capacities if frequent and accurate feedbacks are required. In a shared resource environment this may induce larger delays, that might prevent meeting real-time constraints, while contextual information losses might prevent meeting safety constraints. Integrated design of channel coding and control algorithms is discussed in, e.g., [4]. An approach to jointly design control algorithms and the underlying communication network has been recently devised in [5], where the problem has been cast according to a cross-layer paradigm that combines physical layer, media access control (MAC) layer and control application. Modelling the various interacting components is not trivial even in simplified contexts, while it appears challenging if we also want to look at the wireless network as a useful ubiquitous computing resource for processing and decision: for example, distributed source coding and network coding can be intended as parts of novel computing paradigms that arise in the devised networking context.

A close link between communication and control also arises when we consider that control functionalities are omnipresent in communication systems, with critical examples such as the power control algorithms in cellular systems and the transport control protocol (TCP) in the Internet. In general, any modern communication system, that is targeted to provide a multitude of services, requires adequate control of its communication resources. The problem is exacerbated if we consider that end-to-end communications may often require inter-working among heterogeneous networks (e.g., wireless and wired), wherein the concept of ambient networks for coordinating control functionalities in different transport networks is currently emerging. Especially in the wireless context, where the scarce availability of spectrum slots forces us to handle resource sharing in the access portion of the network, development of effective techniques for management of network resources is recognized at least as important as the development of new transmission techniques that can counteract the hostile propagation channel and increase channel capacity (e.g., advanced channel coding and error recovery mechanisms, modulation techniques and diversity schemes). 
In fact, ultimate achievable spectral efficiency depends on efficient use of resources (e.g., assignment of codes to users and base stations, power levels, coverage handling through efficient beam-forming) that impact on the interference amount that each user signal has to counteract. Although the evident relevance of these control and scheduling problems, many of the mechanisms have not been designed using a model-based control framework, but merely heuristics and ad-hoc solutions. When designing new communication protocols it is of fundamental importance to be able to assess the benefit of also transmitting status information related to the data transmission. In view of the increased system complexity this type of protocols imply, questions such as what information should be transmitted and the quantization of the gain, e.g., in terms of traffic predictability and reliability, needs to be addressed. These are core issues in any network communication system and they are today being far from well understood. It is well known in control theory that old feedback information is of little use; on the contrary it tends to destabilize the system. The implication of this is that status information in a network is perishable and the influence of time delays is an important issue. Control theory has proven to be a suitable framework to analyze such aspects from a systems perspective.

A common need of the two facets of control in communication systems depicted above consists in (i) developing sound modelling of complex systems and environments and (ii) subsequently find suitable optimization and control strategies. Specifically, as it will be remarked throughout the examples, hybrid systems theory may intrinsically provide the mathematical basis for modelling the dynamics of our control systems. While the suitability of such models have been proven and exploited recently in, e.g., the automotive domain, only few and limited attempts (e.g., [6] and [7]) can be found in the literature for communication systems and protocols. Therefore, in this paper we intend to emphasize how hybrid dynamics may actually arise in problems related to operation of communication systems. Specifically, we focus on wireless systems and provide some details on power control in interference-limited fading wireless channels and the behaviour of TCP over a wireless interface.

The outline of the paper is as follows. In Section II we briefly discuss the importance of layered architectures of communication and control systems. We review the layered vision of the OSI model and discuss relations between so called cross-layer optimization and a hybrid systems approach. In Section III we deal with two specific examples, and provide the guidelines for interpreting their hybrid nature. Finally, conclusions and future perspectives are provided in Section IV.

\section{LAYERED ARCHITECTURES FOR COMMUNICATION AND CONTROL}

In the design of large-scale systems, it is crucial to have a design approach based on composition and modularity. This helps the designer to argue about the system and understand

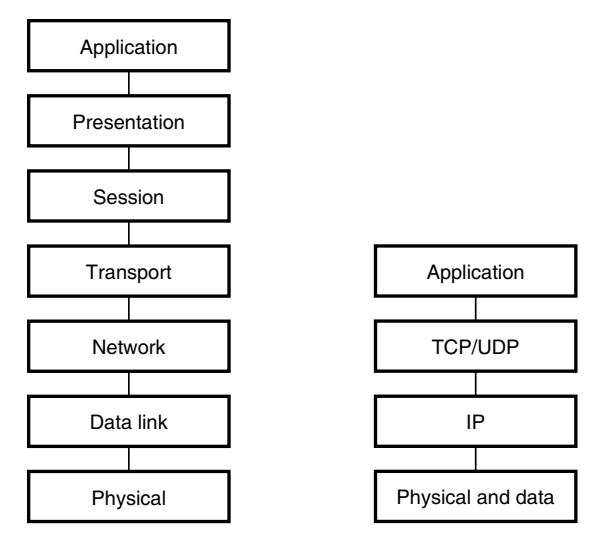

Fig. 1. The layered OSI model for communication networks to the left and the special instance of the Internet to the right.

interactions and dynamics. Layered system architectures are common in many disciplines and widely used in practice. It is surprising that there is not much theory that supports the use [8]. An area that has gained tremendously from a standardized architecture is communication networks. The architecture is an important contributor to the Internet revolution. Here we briefly discuss the OSI model for communication networks and a model for hierarchical control.

\section{A. Communication Networks}

The OSI reference model is shown to the left in Figure 1, see [9], [10] for details. The model is decomposed of seven layers with specified network functions. The lowest layer is the physical layer, which is concerned with transmission of signals from a transmitter to a receiver across a physical medium. Choice of the modulation format is a typical aspect of the physical layer. The data link layer adds error correction on bit level to the unreliable pointto-point communication provided by the physical layer. The main function of the network layer is routing, i.e., to find out where to send packets (sequences of bits). This is typically done by appending an address field to the packet. The transport layer handles messages. It forwards the messages between certain ports of the computers. The session layer sets up sessions between the computers, so that information can be exchanged. The presentation layer makes sure that the syntax used in different computers are translated and it also handles encryption and decryption. Finally, the application layer provides high-level functions needed for the user applications, e.g., file transfer. For the Internet architecture it is common to group some of the OSI layers together. The layered architecture of the Internet is shown to the right in Figure 1. The top three OSI layers have been merged into one. The transport layer is based on either the transport control protocol (TCP) or the user data protocol (UDP). The network layer is defined by the Internet protocol (IP).

Hybrid models are closely related to layered system architectures. The choice of mathematical modelling framework used in communication networks depends obviously 


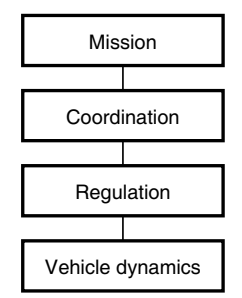

Fig. 2. A layered control architecture for a multi-vehicle control system.

on the purpose of the model. One way of classifying models is by linking them to layers of the OSI model. Models for the physical layer should capture radio signal propagation, interference, modulation etc; models corresponding to the data link layer are of information theoretic character; etc. Cross-layer design is an intensive area of development for particularly wireless networks. When two or more layers are considered, it is natural to be faced with a mixture of model classes. As an example, consider a continuous flow modeling the data transmission of the transport layer. It might be convenient to use such an abstraction, even if data in reality is transmitted as finite messages at discrete instances of time. Routing decisions are of event-triggered nature and may depend on network changes or competing traffic. Hence, to analyze traffic flow over individual links, we might end up with a model having a hybrid nature with a mixture of time-triggered (continuous) dynamics and even-triggered (discrete) dynamics. For further discussion on such a model for TCP, see [7], where the hybrid nature of TCP itself is also investigated. In Section III, we discuss a related model for TCP over wireless systems. It has recently been pointed out that caution needs to be taken in introducing new cross-layer mechanisms [11]. In understanding the interactions such mechanisms may lead to, a rigorous modeling framework is important.

\section{B. Hierarchical Control}

Hierarchical architectures are common also in many control applications, such as in air-traffic management, distributed process control systems, intelligent vehicle highway systems, mobile robotics etc. An example of a layered architecture for a multi-vehicle control system is shown in Figure 2, cf., [8], [12]. The bottom layer consists of the open-loop vehicle dynamics. The second layer is a set of local feedback control laws that regulate the vehicle dynamics, i.e., based on local sensor information provide the vehicle actuators with suitable control commands. The regulation layer provides the coordination layer with a set of maneuvers (e.g., goto way-point, hold maneuver, follow vehicle). The mission layer supervises a team of vehicles by giving each of them sets of maneuvers to execute. The mission layer handles also inter-vehicle communication and error recovery.

For synthesizing controllers and verifying designs, it is useful to employ a hybrid systems framework for hierarchical control systems. Indeed, a major the motivation for developing hybrid systems theory comes from modeling hierarchical control systems [8]. As an example, suppose the lowest layer of the architecture in Figure 2 can be modelled as the open-loop system

$$
\begin{aligned}
& \dot{x}=f(x, u) \\
& y=h(x),
\end{aligned}
$$

where $x$ represents the state of the vehicle (position, heading, etc.), $u$ the controls (steering, throttle, etc.) and $y$ the sensor signals. The regulation layer might be given as

$$
u=c_{k}(y, r),
$$

where $c_{k}$ represents a family of (possibly dynamic) controllers indexed by $k$, and $r$ reference values and other external variables affecting the controls. Both $k$ and $r$ depend on the maneuver imposed by the coordination layer, e.g., for a goto maneuver $c_{k}$ could correspond to the implementation of a time-optimal controller and $r$ the waypoint. The coordination layer is conveniently modelled as a discrete-event system, for which each state corresponds to the execution of a maneuver. Transition takes place either if a maneuver is completed or some other task is given by the mission layer. The integration of the three lower layers of the multi-vehicle control system is hence suitable to model as a hybrid system.

An important extension to the simple hierarchical control model discussed here is the corresponding information and sensing hierarchy. In a networked embedded system, the interaction between control actuation and sensing and information processing is crucial. Under many circumstances, sensing and information processing might be done independent of control (e.g., consider a surveillance robots utilizing a building automation system). This is a conceptually more intrigue system to handle and these are not explicitly captured by the hierarchical control model.

\section{ILLUSTRATIVE ExAMPLES}

In this section two application examples on wireless communication are presented.

\section{A. Power Control}

When considering interference-limited wireless systems, link performance is mainly determined by the signal-tointerference ratio (SIR) statistics. Random channel fluctuations and interfering signals ultimately determine link performance. This is especially true for those systems that are based on direct sequence/code division multiple access (DS/CDMA), in which user signals are allowed to overlap both in time and in frequency, being only distinguishable through spreading and scrambling codes. DS/CDMA is a basic access technique for the radio interface of third generation wireless systems, e.g., in so called W-CDMA and CDMA2000. These systems have been defined for supporting heterogeneous traffic, with a variety of source rates and quality of service requirements. The achievement of large capacities and adequate performance in this context 


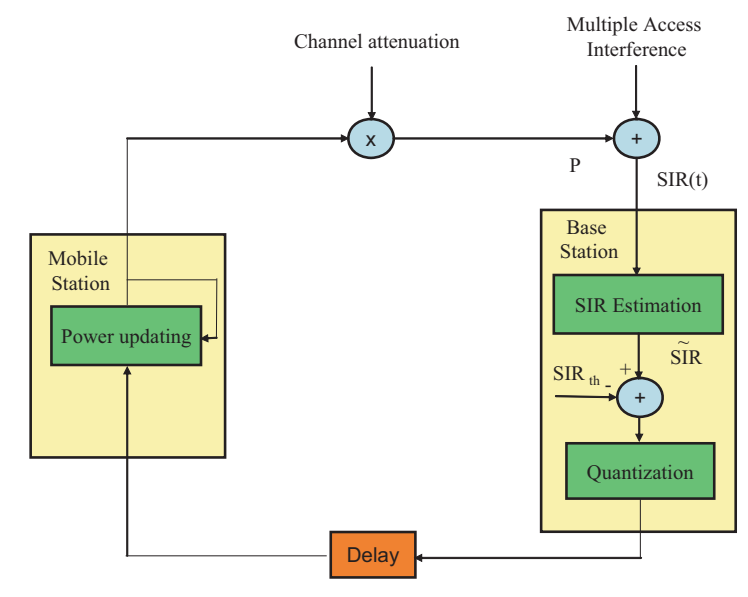

Fig. 3. Power control of third generation wireless system. The closedloop control system has hybrid dynamics in that there is a mixture of time-triggered and event-triggered signals, and the communicated control command is quantized.

is a challenging task, and requires a proper allocation of system resources. Moreover, as the environment is timevarying, adaptive transmission techniques are envisaged, with various combinations of alternatives for power and rate allocation, coding formats, error recovery mechanisms, and so on.

Among various techniques, power control is an essential functionality to combat the near-far effect and let each user achieve its target SIR at every time. Apart from the open loop component, in modern systems there is a closed-loop control. It consists of an outer loop and an inner loop. The outer loop adapts the target SIR based on link quality estimation, while the inner loop is responsible for power adaptation in order to meet the target SIR. Let us consider the reverse link in a multi-user system, i.e., mobile station (MS) to base station (BS). The closed-loop acts for each user signal, so that there is a set of interacting loops, each one acting as follows, see Figure 3. At each symbol time, an estimation of the SIR is performed e.g. with an averaging filter over a block of $B$ symbol intervals and compared to the target level. Thus, a new estimate of the SIR is available at the filter output every $B$ symbol time interval. The difference between the filter output and the target level is then used to decide which is the power correction to be applied at the MS. Specifically, such a difference is quantized in order to meet the available bandwidth for the power update command to send on a forward (BS to MS) link power control channel. For example, binary quantization could be used, where either the bit 1 is sent to increase the MS transmission power, or the bit 0 is sent to decrease the power. After a delay, due to propagation and processing, the command is received by the MS. The new transmitted power at the MS is obtained by applying the correction to the last transmitted power level. The transmitted power is kept constant until a new update command is received. A well founded view of power control is provided in [13].

The power control system described above is obviously a quantized feedback system. Hybrid dynamics also arise from that the target SIR updates are events that take place on a larger time scale with respect to regular (synchronous) transmission power updates forced by the inner loop. Moreover, power control can not be considered alone in the adaptive transmission context we have envisaged. In fact, rate adaptation among a limited set of alternatives is allowed and jointly combined with target settings in the outer loop. In addition, adaptive coding formats also interact with power control and contribute to define the event-based component of the hybrid system. An attempt to model the complexity of interactions among all these components has been proposed in some recent papers [14], [15]. In particular, in [14] a model is derived for the powercontrolled and interference-limited wireless channel, and then evaluation of performances of forward error correction (FEC) and hybrid automatic repeat request (ARQ) error control coding is performed over the abstracted channel model.

\section{B. TCP/IP over Wireless Systems}

A sound layered communication architecture is important, e.g., [11]. The tremendous growth of the Internet is to a large extent due to the architecture illustrated in Figure 1. New technology and cross-layer algorithms may, however, challenge the separation of the layers. One example is given by wireless Internet, in which there are one or more wired links replaced by radio transmissions. In this case, as is shown below, the physical and data link layer may influence upper layers and thereby deteriorate performance.

Consider a single user that connects to the Internet through a mobile terminal. An illustration of the system is shown in Figure 4, where four interacting feedback control loops are indicated. At the lowest level, the transmission power is controlled in order to keep the SIR at a desired level, as discussed in previous sub-section. This is a fast inner loop (1) intended to reject disturbances in the form of varying radio conditions. On top of this, we have an outer power control loop (2) that tries to keep the frame error rate constant, by adjusting the target SIR of the inner loop. Next, we have a local link-layer retransmission of damaged radio frames through the automatic repeat request mechanism (3). Finally, the end-to-end congestion control of TCP (4) provides a reliable end-to-end transport for the application with built-in flow control.

Cross-layer interactions may reduce the end-to-end throughput. For the wireless Internet scenario introduced above, the two nested power control loops are supposed to support the separation of the physical layer from the data link layer. The automatic repeat request should separate the data link layer from the network layer. TCP should separate the transport layer from the application by providing a virtual end-to-end connection between the mobile terminal and the Internet server. A timeout event in TCP occurs 


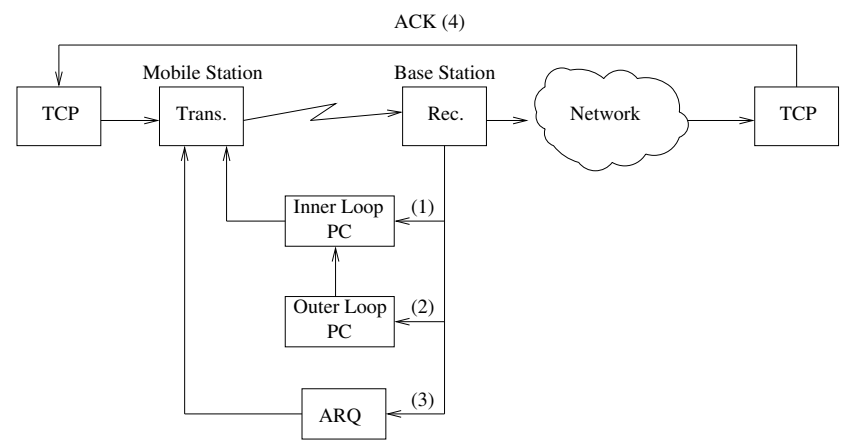

Fig. 4. System overview of wireless Internet in a case when a mobile user connects to an Internet server through a TCP/IP session. Four of the feedback control loops that support the separation of the layers in the network architecture are indicated: the inner power control loop (1), outer power control loop (2), link-layer retransmission (3), and end-to-end congestion control (4). See [16] for details.

when a packet, or its acknowledgement, is delayed too long. The timeout mechanism is supposed to indicate severe congestion and thereby force TCP to reduce the sending rate drastically. Spurious timeouts, i.e., timeouts that are not due to network congestion, are known to sometimes occur if the lower layers are not working properly [17]. It was recently shown that realistically modelled radio links influence the delay distribution of the TCP segments and that they induce spurious timeouts [16]. The performance degradation measured in throughput can be up to about $15 \%$. The analysis is based on a hybrid model derived from Figure 4, where the power control loops are modelled through a Markov chain. The influence of a more detailed radio model was studied in [18].

There are a few proposals to improve TCP performance over radio links. One is to change the TCP algorithms to make them more robust to link irregularities, e.g., [19]. Another is to engineer the link-layer, to give it properties that plain TCP handles well. In view of the discussion above on that caution needs to be taken in introducing new crosslayer mechanisms, it is not always desirable to optimize one layer of the network architecture for a specific application or operating condition. Another drawback with modifying TCP algorithms is that deployment of new algorithms affect all Internet end systems, which makes it a slow and costly process. Tuning the link properties is more practical from a deployment point of view, at least if the tuning can be done before widespread adoption of a new link type. If possible, the radio links should be made as friendly as possible to a large class of data traffic [16]. The fundamental limitations need to be investigated of the system. It was shown in [20] that without any cross-layer signalling, the delay distribution could in a very simple way be adjusted by adding a suitable delay to certain TCP segments and thereby gain considerably improvements of the throughput. The design and implementation of new hybrid controller for improved user experience of wireless Internet was discussed in [21]. Information on radio bandwidth and queue length available in the so called radio network controller (RNC), close to

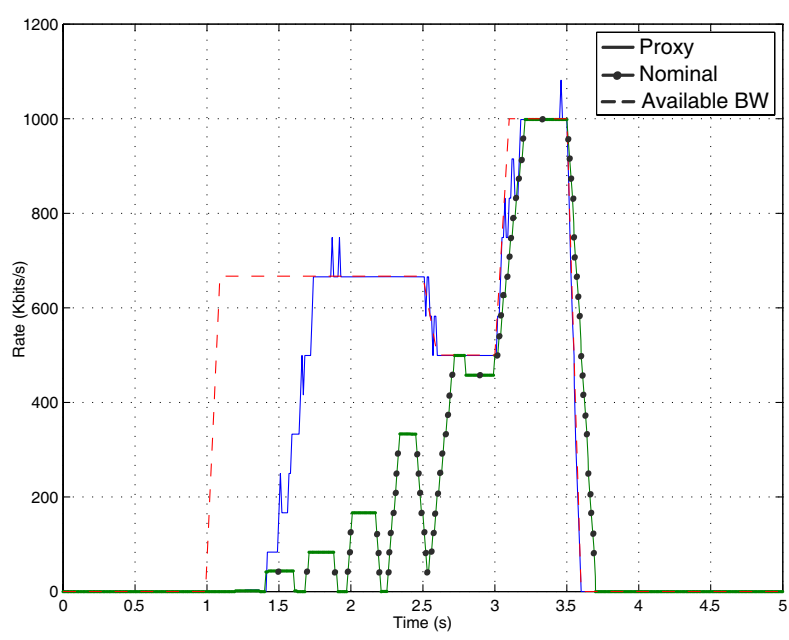

Fig. 5. Improved TCP over a wireless link through a new proxy-based hybrid controller. Available bandwidth (dashed) over the wireless link, compared to the actual utilization for the proxy setup (solid) and the nominal setup (dotted). See [21] for details.

the base station, is used in a proxy that resides between the Internet and the cellular system. The hybrid control algorithm in the proxy sets the window size according to event-triggered information on radio bandwidth changes and time-triggered information on the queue length of the RNC. Figure 5 illustrates a typical improvement of bandwidth utilization of the proxy setup compared to the nominal setup today. The available bandwidth of the wireless link is shown by the dashed line. The bandwidth variations are due to the varying conditions of the radio link. The dotted line shows the utilization for the nominal setup with TCP Reno (without proxy). The solid line shows the utilization with the new proxy solution. Note that none of the setups achieves full link utilization, but the proxy setup tracks the bandwidth variations much better than the nominal setup, and it adjusts more quickly to the available bandwidth. This results in faster response and better utilization of the radio link. The initial delay of about 0.4 seconds corresponds to the TCP connection establishment, and affects both setups equally. The oscillations in the nominal setup is a result of the bursty behavior of the Slow Start mechanism of standard TCP. See [22] for a control theoretic analysis of this hybrid control scheme.

\section{CONCLUSIONS}

Through some application examples, we have illustrated the the importance of a research agenda on the convergence between communications and control. Specifically, we have described how some relevant control problems in wireless communications could be usefully cast in terms of hybrid systems for consistent modelling of significant interactions. Current research work is progressing along the two main tracks of control of networks and control over networks, with specific interests on various aspects of distributed radio resource management in evolved third generation wireless 
systems, and efficient design and operations of ad-hoc wireless networks for control applications.

\section{ACKNOWLEDGEMENTS}

The research agenda outlined in the paper forms the basis for a workpackage on networked control in the EU FP6 Network of Excellence HYCON. Contributions by the partners are gratefully acknowledged. The work presented in Section III is based on joint work with colleagues and students at KTH and DEWS.

\section{REFERENCES}

[1] D. Estrin, Ed., Embedded, Everywhere: A Research Agenda for Networked Systems of Embedded Computers. U.S. National Research Council, 2001.

[2] R. M. Murray, Ed., Control in an Information Rich World: Report of the Panel on Future Directions in Control, Dynamics, and Systems. SIAM, 2003.

[3] S. Graham, G. Baliga, and P. Kumar, "Issues in the converging of control with communication and computing: Proliferation, architecture, design, services, and middleware," in Proc. 43rd IEEE Conference on Decision and Control, 2004.

[4] S. Mitter, "Control with limited information: the role of systems theory and information theory," IEEE Information Theory Society Newsletter, vol. 50, 2000, iSIT 2000 Plenary Talk.

[5] X. Liu and A. Goldsmith, "Wireless network design for distributed control," in Proc. 43rd IEEE Conference on Decision and Control, 2004.

[6] T. Alpcan and T. Basar, "A hybrid systems model for power control in multicell wireless data networks," Performance Evaluation, vol. 57, pp. $477-495,2004$.

[7] J. Hespanha, S. Bohacek, K. Obraczka, and J. Lee, "Hybrid modeling of TCP congestion control," in Hybrid Systems: Computation and Control, ser. Lecture Notes in Computer Science, M. di Benedetto and A. L. Sangiovanni-Vincentelli, Eds. Springer-Verlag, New York, 2001, vol. 2034.

[8] P. Varaiya, "Smart cars on smart roads: Problems of control," IEEE Transactions on Automatic Control, vol. 38, no. 2, pp. 195-207, 1993.

[9] J. D. Day and H. Zimmermann, "The OSI reference model," Proceedings of the IEEE, vol. 71, pp. 1334-1340, Dec. 1983.

[10] J. Walrand and P. Varaiya, High-performance communication networks, 2nd ed. Morgan Kaufmann, 2000.

[11] V. Kawadia and P. R. Kumar, "A cautionary perspective on crosslayer design," IEEE Wireless Communications, vol. 12, no. 1, pp. $3-11,2005$.

[12] J. de Sousa, K. H. Johansson, A. Speranzon, and J. Silva, "A control architecture for multiple submarines in coordinated search missions," in IFAC World Congress, Prague, Czech Republic, 2005, to appear.

[13] F. Gunnarsson and F. Gustafsson, "Power control in wireless communications networks-from a control theory perspective," in IFAC World Congress, 2002.

[14] F. Babich, F. Santucci, and F. Graziosi, "Modeling of powercontrolled interference-limited wireless channels," in WPMC. IEEE, 2002.

[15] F. Santucci, G. Durastante, F. Graziosi, and C. Fischione, "Power allocation and control in multimedia cdma wireless systems," Kluwer Telecommunication Systems, vol. 23, pp. 69-94, 2003.

[16] N. Möller and K. H. Johansson, "Influence of power control and linklevel retransmissions on wireless TCP," in Quality of Future Internet Services, ser. Lecture Notes in Computer Science. Springer-Verlag, 2003, vol. 2811.

[17] R. Ludwig and R. H. Katz, "The eifel algorithm: Making tep robust against spurious retransmissions," ACM Computer Communication Review, vol. 30, no. 1, January 2000.

[18] C. Fischione, F. Graziosi, F. Santucci, N. Möller, K. H. Johansson, and H. Hjalmarsson, "Analysis of TCP over WCDMA wireless systems under power control, MAI and link level error recovery," in $I W C T, 2005$.

[19] S. Mascolo, C. Casetti, M. Gerla, M. Y. Sanadidi, and R. Wang, "TCP Westwood: bandwidth estimation for enhanced transport over wireless links," in MobiCom, Rome, Italy, 2001.
[20] N. Möller, K. H. Johansson, and H. Hjalmarsson, "Making retransmission delays in wireless links friendlier to TCP," in Proc. 43rd IEEE Conference on Decision and Control, 2004.

[21] I. C. Molero, N. Möller, J. Petersson, R. Skog, Å. Arvidsson, O. Flärdh, and K. H. Johansson, "Cross-layer adaptation for TCPbased applications in WCDMA systems," in IST Mobile and Wireless Communications Summit, Dresden, Germany, 2005.

[22] N. Möller, I. C. Molero, K. H. Johansson, J. Petersson, R. Skog, and Å. Arvidsson, "Using radio network feedback to improve TCP performance over cellular networks," in IEEE Conference on Decision and Control and European Control Conference, Seville, Spain, 2005, invited paper. 